Annales de Toxicologie Analytique, vol. XII, $\mathrm{n}^{\circ}$ 1, 2000

\title{
Les produits dopants de demain
}

\section{The doping products of tomorrow}

Laurent RIVIER

Laboratoire Suisse d'Analyse du Dopage, Institut Universitaire de Médecine légale, rue du Bugnon 21, CH-1005 LAUSANNE - SUISSE - Tél : +412213147330 - Fax : +41213147333 e-mail : Laurent.Rivier@inst.hospvd.ch

(Reçu le 16 novembre 1999 ; accepté le 8 janvier 2000)

\section{RÉSUMÉ}

Les techniques de dopage chez les sportifs se sont développées de façon spectaculaires ces dernières années. Les amateurs de produits ergogènes ont recours à une imagination débridée, basée sur des similitudes grossières entre action pharmacologique moléculaire et physiologie de l'organisme entier. Ils mettent ainsi leur santé, voire parfois même leur vie en jeu en utilisant, par exemple, des produits pharmaceutiques non testés en clinique pour gagner quelques fractions de seconde sur leurs adversaires. Les laboratoires officiels de dépistage du dopage mettent en cuvre pour l'instant des stratégies basées uniquement sur l'analyse d'urines prélevées après les compétitions ou pendant l'entraînement. Les résultats de ces contrôles peuvent paraître dérisoires car certaines substances indétectables - et qui figurent sur la liste des produits prohibés - sont largement utilisées. Sur le plan de la recherche de la performance optimale au moyen de substances défendues non détectables, le risque de se faire prendre aux contrôles est donc aujourd'hui nul. En conséquence, le recours à des nouvelles molécules n'apparaît pas être une nécessité première. Cependant, l'athlète tricheur qui disposera seul d'un produit inédit, bénéficiera d'un avantage certain, jusqu'au moment où cette pratique sera connue d'un trop grand nombre de concurrents. Il y a donc surenchère dans ce domaine. Les laboratoires de dépistage sont attentifs à l'évolution de ces pratiques. De nouvelles stratégies analytiques se mettent en place, certaines basées sur l'analyse du sang et sur la notion de suivi biologique. Les recherches menées pour la détection de l'abus d'Erythropoïtine et d'Hormone de croissance en sont l'illustration la plus actuelle. D'autres produits issus de la

\section{SUMMARY}

New techniques in sport doping have emerged the last few years. Most of them are based on gross similarities between molecular pharmacological action and whole organism physiology. Most often new chemicals are used without waiting the safety coming from the ordinary clinical tests. Athletes are taking such high health risks for gaining a few seconds on their competitors. On the detection side, laboratories are working on urines collected after competition or during training. Globally, the number of positive findings from laboratories seem low in regard to the wide use of the undetectable compounds, which are already on the list of forbidden substances. The risk to be detected after using such kind of chemicals at an official control is nonexistent. Consequently, it seems that the necessity for a recourse to use new substances is rather low. However, the athlete who could use first a newly discovered pharmacological agent will get a definite advantage over his competitors until these latter are too numerous in using the same product as well. Laboratories for doping detection are aware of that and introduce new strategies based on blood analyses and individualsi biological following up. Recent researches aiming for the detection of abuse of Erythropoietin and human Growth Hormone are good illustrations of that. Other products originating from organic synthesis like the PFCs or from recombinant technologies like IGF-1 or Interleukin-3 are more often cited in the medias without any formal indication of their use. Mixtures of small doses of anabolic steroids or subcutaneous implants for the slow release of active substances seem to be in actual use as well as curare-like synthetic compounds. New instrumental strategies based on GC-C-IRMS or LC-MS/MS, for 
synthèse chimique organique, tels les PFCs ou de la biotechnologie recombinante, telles l'IGF-1 ou l'Interleukine-3, sont de plus en plus cités dans les médias, sans que la preuve de leur utilisation ait été réellement établie. On parle aussi de mélanges à petites doses de stéroüdes anabolisants, d'implants sous-cutanés pour le re-largage lent des substances actives, ou même de produits aussi exotiques que les curarisants dont on ne conçoit que difficilement l'emploi dans le cadre de la performance sportive. Le rôle des laboratoires est de travailler sans relâche à la découverte de nouvelles molécules dans les échantillons biologiques auxquels ils ont accès. Les stratégies analytiques choisies font appel aux technologies instrumentales les plus récentes (Chromatographie en phase gazeuse couplée à la détermination en ligne de rapports isotopiques du Carbone par spectrométrie de masse : GC-C-IRMS, ou Chromatographie liquide à haute pression couplée à la spectrométrie de masse tandem : LC-MS/MS, etc.). Mais la détection formelle d'une nouvelle molécule n'est pas suffisante en elle-même pour caractériser le dopage : l'aspect juridique de la question ne pourra opérer qu'une fois la procédure validée par les scientifiques. Dans l'intervalle, l'attribut de dopage sportif devra être encore enregistré par le C.I.O. et par toutes les associations sportives internationales. Si le parcours semble rébarbatif, le défi est déjà relevé par bon nombre des laboratoires de dépistage dans le monde.

\section{MOTS-CLÉS}

Dopage sportif, dépistage analytique, nouvelles substances, analyse instrumentale.

\section{Introduction}

Le dopage n'est pas un phénomène nouveau dans le monde sportif, mais ce qui change aujourd'hui c'est son développement au niveau mondial dans un contexte d'exaltation de la réussite individuelle et de la marchandisage croissante du sport. Pour retirer le plus de profits possible, les nouveaux entrepreneurs du sport cherchent à retirer des rendements de plus en plus élevés et le sport de compétition est devenu un véritable business : création de sociétés commerciales, introduction de clubs en bourse, prise de participation dans leur capital par de grandes firmes multinationales, privatisation croissante du financement du spectacle sportif... (1). Derrière ce marché, s'est développée parallèlement l'industrie du dopage dont il est impossible d'évaluer aujourd'hui l'étendue de par le silence entretenu par les parties intéressées en premier chef à préserver l'image même du sport de haute compétition. Or, la demande de produits dopants de par le monde ne cesse de se développer. Du retour sur investissement attendu par les nouveaux entrepreneurs du sport de compétition découle l'obligation du sportif à la performance et au bon résultat sous peine de sanctions immédiates : la relégation, la perte du salaire et l'oubli. Le régime intense des compétitions choisi pour alimenter le spectacle du sport "fabrique" des athlètes qui vont se fatiguer prématurément. Ces paramètres associés encore à d'autres liés à notre culture de compétition dans l'en- example, are implemented by laboratories. But formal characterization of doping lays not just on the detection of a defined molecule, the juridical aspects will also be covered after all international sport associations as well as the I.O.C. have duly registered it. The way to success for the detection. of all these new chemicals is long but the analytical challenge is important.

\section{KEY-WORDS}

Sport doping, analytical strategy, new substances, instrumental analysis.

semble de la société débouche sur une consommation beaucoup plus large de produits aidant la performance. Quelques chiffres d'estimation des coûts liés au dopage sportif donnent un éclairage particulièrement instructif sur l'ampleur du phénomène et le déséquilibre patent entre les moyens mis en cuvre pour la lutte contre ce fléau et les ressources utilisées pour modifier la performance sportive. Espérons que l'Agence Mondiale Antidopage, nouvellement créée à Lausanne va permettre de renverser cette tendance rapidement.

Tous les produits dopants qui figurent sur la liste du C.I.O. ne sont pas illégaux et il existe une série de produits licites utilisés pour permettre de tenir le coup. Un vaste marché s'est développé qui est constitué de produits ergogènes licites, médicaments préparateurs ou reconstituants autorisés (2). Parmi les produits interdits mais utilisés en médecine en dehors du sport, on sait que la justification thérapeutique ne couvre qu'une faible partie de ce qui est produit et vendu officiellement par les fabricants. Ainsi les révélations du sprinter canadien Ben Johnson en 1988 sur l'utilisation du Stanozolol ont certainement fait plus pour la publicité de cet anabolisant que n'importe quelle campagne. Après les résultats mirifiques du joueur de base-ball Mark McGwire dix ans plus tard obtenus par la prise de l'androstènedione, les ventes de ce produit interdit par le C.I.O., mais classé comme complément nutritionnel aux U.S.A. par la "Food and Drug Administration", ont augmenté de $150 \%$. 
Tableau I : Comparaison des cô̂ts respectifs du contrôle anti-dopage officiel, des ressources de recherche et des moyens financiers inofficieux qui seraient mis à disposition pour le dopage sportif, listés par ordre croissant des sommes impliquées (adapté de 1 et complété).

\begin{tabular}{|l|l|}
\hline Domaine concerné & Coûts \\
\hline Contrôle antidopage (un prélèvement et une analyse d'urine) & 500 Euro \\
Traitement d'Epo sur une saison pour un coureur cycliste de faible niveau international & 8000 Euro \\
Cure mensuelle de diurétiques et d'anabolisants pour un culturiste de haut niveau & 15000 Euro \\
Traitement annuel en hGH et Epo pour un champion cycliste & 50000 Euro \\
Estimation du budget annuel consacré au dopage par une grande équipe cycliste & 100000 Euro \\
Échelle des revenus annuels des médecins du sport & 0,15 à 1,5 Mio Euro \\
Subsides de recherche octroyés par le C.I.O. sur l'Epo et la GH pour l'an 2000 & 2 Mio Euro \\
Budget de la lutte antidopage du ministère français de la Jeunesse et des Sports & 2,3 Mio Euro \\
Estimation du budget de la lutte antidopage dans le monde & 60 Mio Euro \\
Ventes mondiales de créatine (estimation) & 100 Mio Euro \\
Ventes mondiales de stéroïdes anabolisants (estimation) & 1000 Mio Euro \\
Chiffre d'affaire global du dopage dans le monde (sport de haut niveau) & 4000 à 8000 Mio Euro \\
\hline
\end{tabular}

\section{Difficultés de la lutte anti- dopage}

Le développement de la demande, de même que les progrès de la pharmacologie, ont donné aux produits dopants un intérêt morbide qui font que nous hésitons souvent à donner toutes les informations spécifiques auxquelles les laboratoires officiels de dépistage du dopage pourraient avoir accès. Dans le même ordre d'idée, il faut bien reconnaître que ces laboratoires seront les derniers à être informés spontanément de l'utilisation nouvelle de telle ou telle substance. Nous avons donc dû établir nos propres réseaux d'informations de façon à rester au courant des tendances les plus récentes dans ce domaine. Nous avons pu constater avec nos collègues des laboratoires accrédités antidopage que de nouvelles substances et préparations appa- raissaient et que de nouvelles méthodes étaient mises en œuvre pour contourner les directives en se basant sur le précepte : tout ce qui n'est pas interdit, est utilisable. Des progrès certains ont été obtenus dans la rapidité d'adaptation des laboratoires et des mouvements associatifs sportifs pour suivre les nouvelles tendances (cf. Tableau 2). De plus, et c'est un nouvel éclairage très inquiétant, diverses études épidémiologiques et les quelques enquêtes récentes effectuées sur le terrain ont montré sans ambiguîté qu'il y avait une similitude certaine entre le développement des habitudes du dopage et celui de la scène de la drogue : le recours à des circuits inofficieux de distribution, des produits de qualité incertaine et de provenance douteuse, des prix largement surfaits par rapport à ceux pratiqués par l'industrie pharmaceutique et, enfin, l'utilisation de doses largement disproportionnées par rapport à une éventuelle indication thérapeutique de la médecine officielle.

Tableau II : Illustration de la difficulté de la lutte anti-dopage dî au temps considérable nécessaire à caractériser chimiquement, juridiquement et réglementairement l'infraction de dopage (adapté de (3) et complété).

\begin{tabular}{|lll|}
\hline Produits dopants & $\begin{array}{l}\text { Première utilisation sportive } \\
\text { reconnue }\end{array}$ & $\begin{array}{l}\text { Nombre d'années écoulées entre } \\
\text { la première utilisation sportive et } \\
\text { le premier détecté officiellement }\end{array}$ \\
\hline Amphétamines & 1936 & 32 ans \\
Anabolisants & 1954 & 22 ans \\
Corticoïdes & $1960 ?$ & $\begin{array}{l}\text { Décelables dès 1980 environ mais pas } \\
\text { recherchée systématiquement }\end{array}$ \\
Diurétiques & 1968 & 18 ans \\
Bêta-bloquants & 1978 & 7 ans \\
Hormone de croissance & $1980 ?$ & Indécelable \\
Epo & $1987 ?$ & Indécelable \\
Immunostimulants (exemple : Bromantane) & 1994 & 3 ans \\
\hline
\end{tabular}




\section{Molécules dopantes exis- tantes}

Il n'existe pas de liste exhaustive qui contiendrait toutes les substances qui ont la capacité de modifier les performances sportives. La définition même du Comité International Olympique (C.I.O.) éclaire mieux la notion de dopage depuis la parution du nouveau "Code antidopage du mouvement olympique" (4). La liste d'exemples qui y figure est modifiée en début de chaque année. Ces modifications portent, soit sur de nouvelles substances, soit sur les valeurs de concentrations au-delà desquelles un résultat de laboratoire est considéré comme positif. Depuis cette année, il est aussi précisé que certaines substances sont interdites pour les hommes uniquement (clomiphène, cyclofenil, tamoxifen, Gonadotropine chorionique (hCG) et hormone lutéinisante (LH) et ses facteurs de libération, par exemple). Il est aussi précisé que le groupe des corticostéroïdes est maintenant intitulé "gluco-corticostéroïdes". Enfin, il est intéressant de noter également que pour les béta-bloquants, tous ceux figurant dans l'ouvrage de référence intitulé "Martindale, The Extra Pharmacopoeia"(5), sont maintenant inscrits sur la liste d'exemples de substances interdites (6). Ces changements interviennent maintenant dès le début de l'année et doivent donc être pris en considération immédiatement. C'est, en quelque sorte, le baromètre indicateur de l'état officiel des substances dopantes. Cependant, il faut être attentif au fait que cette liste d'exemples de substances interdites n'est pas exhaustive. De nombreuses substances, non répertoriées dans cette liste, sont considérées comme interdites sous l'appellation "substances apparentées" et les athlètes doivent s'assurer que tout médicament, supplément, préparation en vente libre ou toute autre substance qu'ils utilisent ne contient aucune substance interdite. Selon notre collègue, le Professeur Klaus Mueller du laboratoire de Keischia (7), ce seraient plus de 1000 substances reconnues pour agir sur la performance physique, déjà répertoriées dans Ie Mardindale ou l' "Index Nominum, International Drug Directory" (8), deux livres de référence qui donnent toute l'information de base sur les drogues et les médicaments du monde entier, qui seraient concernées. La liste complète de ces substances actives devrait être publiée prochainement sous l'égide du Conseil de l'Europe.

\section{Molécules de demain}

Une remarque préliminaire s'impose. Il est clair que les laboratoires officiels de dépistage du dopage, c'est-àdire les 28 laboratoires actuellement accrédités par le C.I.O.) ne vont pas clamer haut et fort les vertus de nouvelles molécules modifiant les performances sportives. Notre code de déontologie qui figure dans le code antidopage déjà cité (3) est explicite à ce sujet. Nous ne sommes pas autorisés à effectuer des recherches non fondées scientifiquement, ni administrer diverses molécules afin d'étudier l'amélioration des performances d'athlètes de pointe : le cas récent de certaines expériences conduites par le Professeur Conconi en est un exemple. Il avait publié en 1993 des travaux (9) sur l'administration d'Epo à des athlètes pour étudier l'effet de cette hormone et déterminer s'il était possible d'en détecter l'usage dans le sang. Aujourd'hui, près de 10 ans après ces expériences, les circonstances dans lesquelles elles ont eu lieu ne semblent pas assez claires pour que la justice italienne ait décidé de lui demander des explications approfondies...

Les noms de molécules ou de méthodes qui sont indiqués dans cet article ont tous été évoqués préalablement, soit dans des publications sérieuses à caractère public avisé, soit dans des revues de vulgarisation, ou enfin, dans quelques articles de quotidiens. Avec bon nombre de mes collègues, je suis convaincu qu'il existe un certain nombre de laboratoires non accrédités, certains oeuvrant en parfaite ouverture alors que d'autres agissent malheureusement plus ou moins clandestinement, mettent au point des procédures alternatives et/ou complémentaires de dépistage ou même étudient des régimes dopants inédits. Malheureusement les publications scientifiques originales sur le sujet restent rares. Paradoxalement nous observons l'intérêt général soutenu qui s'est développé ces trois dernières années pour tout ce qui touche au dopage sportif, alors que le nombre de laboratoires accrédités reste réduit pour un nombre de contrôles limité. Rappelons qu'actuellement ces laboratoires effectuent au niveau mondial un peu plus de 150000 contrôles chaque année.

Ces molécules nouvelles peuvent être rangées en 2 catégories principales : les xénobiotiques et les substances dites naturelles. Ces produits ne sont actuellement pas inclus dans les contrôles antidopage, soit parce qu'ils ne sont pas spécifiquement caractérisés, soit que leur détection n'est pas encore possible. En effet, de nombreuses substances dopantes interdites par le C.I.O. (Erythropoïétine (Epo), hormone de croissance humaine (hGH), émultions de perfluorocarbures (PFCs), etc.), connues pour être utilisées par les athlètes, ne seraient décelables que dans le sang et/ou l'air expiré. Comme, jusqu'à ce jour, le C.I.O. n'a pris en compte que les contrôles urinaires pour fixer des sanctions, les dopés riches et donc généralement bien conseillés sortent négatifs relativement facilement aux contrôles officiels. Quant à l'insuline, aux anti-inflammatoires non stéroïdiens et aux glucocorticoïdes, ils sont autorisés sous certaines conditions. La justification thérapeutique qui est exigée lors de l'administration de ces médicaments est tellement facile à produire, que les laboratoires ne déploient pas des prodiges pour les 
détecter sachant que par expérience, les résultats positifs pour ces substances ne seront dans la majorité des cas pas suivis d'effets. Dans le tableau 3 figure la liste des substances connues pour être utilisées et qui posent toujours problème quant à leur détection fiable et systématique par les laboratoires accrédités. Il faut souhaiter que les progrès des recherches en cours permettent de trouver une solution durable à la mise en place de procédés de détection fiable. Il faut aussi que ces nouvelles approches de laboratoire basées, par exemple sur les mesures de marqueurs secondaires, soient suffisamment fiables et robustes pour tenir compte des moyens que je qualifierai de "périphériques" de se doper, comme ce serait le cas pour les "peptides sécrétagogues", par exemple. Nous verrons ci-dessous que plusieurs de ces peptides n'ont pas apportés les résultats attendus en thérapeutique et ont été abandonnés par l'industrie pharmaceutique. La facilité avec laquelle d'autres laboratoires pourraient produire ces même molécules fait penser qu'il serait très possible de les retrouver comme agents dopants.

Chaque produit nouveau correspond à une application particulière dans le sport et présente sa propre problématique lors de sa détection. Nous allons passer en revue ces composés les uns après les autres :

\section{Xénobiotiques}

1. Les Perfluorocarbures (PFCs) sont des molécules totalement synthétiques, correspondant à une sorte de téflon liquide (Figure 1). Ils ont la particularité d'être totalement inertes, de ne pas se métaboliser et de dissoudre des quantités très importantes de gaz. Dans un contexte précis, ils peuvent être utilisés comme transporteurs d'oxygène (12). Si dans le corps l'oxygène est naturellement transporté par l'hémoglobine du sang des poumons aux muscles, les PFCs permettent de multiplier le rendement de ce transport de façon significative si on respire de l'oxygène pur auparavant. Contrairement à l'Epo qui développe ses effets sur l'hémoglobine après 2 à 3 semaines, les PFCs ont une action instantanée. La difficulté majeure de l'administration de PFCs réside en la production d'une émulsion stable qui soit compatible avec le sang (13). La première émulsion injectable de PFCs a été développée dès 1976 au Japon sous le nom de "Fluosol". Cette préparation a pu être utilisée pour assurer l'oxygénation de tissus utilisés pour des transplantations d'organes. Ce produit a été retiré du commerce car il devait être conservé congelé et une préparation complexe était nécessaire juste avant son utilisation. Il n'est pas exclu que certains flacons de ce produit aient été utilisés dans le domaine sportif. Selon des informations non vérifiées, il semblerait que d'autres sources de PFCs pourraient circuler dans les milieux sportifs. La première proviendrait de Chine et la seconde de Russie. Ce der- nier produit s'appelle "Perftoran" et il serait disponible en pharmacie à Moscou pour des indications médicales nécessitant "la correction de types variés d'hypoxie". Un site Internet de la maison qui fabrique le produit ne donne que peu d'informations supplémentaires. Une troisième préparation, sur le point d'être mise sur le marché, nous vient d'Amérique. C'est le laboratoire californien Alliance Pharmaceutic qui a imaginé le produit "Oxygent" dont la particularité est sa relative faible toxicité, sa grande stabilité - il est prêt à l'emploi - et la rapidité de son excrétion de l'organisme. Ce nouveau produit est donc beaucoup plus facile à manipuler que les précédents puisqu'il est stable à température ambiante. Nos tentatives répétées d'obtenir un flacon pour des tests scientifiques ou pour la mise au point d'une procédure analytique ont toutes essuyé un refus catégorique de la compagnie pharmaceutique. Il faut espérer que ce verrouillage subsistera également après que les phases de mise au point soient terminées et que le produit sera disponible dans les hôpitaux.

Il faut noter qu'il est facile de se procurer les PFCs pour tenter de fabriquer son émulsion maison. Cependant, le savoir-faire nécessaire à l'élaboration du produit stérile, stable et compatible avec la circulation sanguine n'est pas à la portée du premier venu. Il est pourtant possible que les recettes de préparations aient été dérobées à l'insu des fabricants, malgré toutes les précautions prises, tant les intérêts financiers sont importants.

La détection des PFCs dans le sang ou l'air expiré ne pose pas de problèmes particuliers aux laboratoires qui disposent de l'équipement adéquat. Le Prof. Michel Audran de la Faculté de Pharmacie de Montpellier a mis au point une méthode basée sur l'extraction par un solvant organique et une analyse par GC-MS (14) alors que nous avons développé une méthode ne nécessitant aucune préparation basée sur l'échantillonnage par espace de tête et analyse automatisée par GC-MS (15). D'autres laboratoires pratiquent ces méthodes sur des échantillons prélevés sur des sportifs en dehors des programmes officiels de contrôles de dépistage du dopage. Ils sont alors effectués dans un contexte général de médecine du travail ou dans le cadre de programmes de prévention de la santé, voire lors d'actions judiciaires ponctuelles.

2. Selon des informations provenant de médecins du sport, il semblerait que certains préconiseraient l'administration probablement par voie intramusculaire de faibles doses de divers relaxants musculaires ou curarisants dont la nature exacte reste encore inconnue. La logique sous-jacente de cette hypothétique pratique n'est pas évidente à mettre en ćvidence par rapport à l'augmentation de la performance sportive. On pourrait imaginer que l'effet recherché consisterait à tenter de diminuer l'apparition de crampes musculaires provo- 


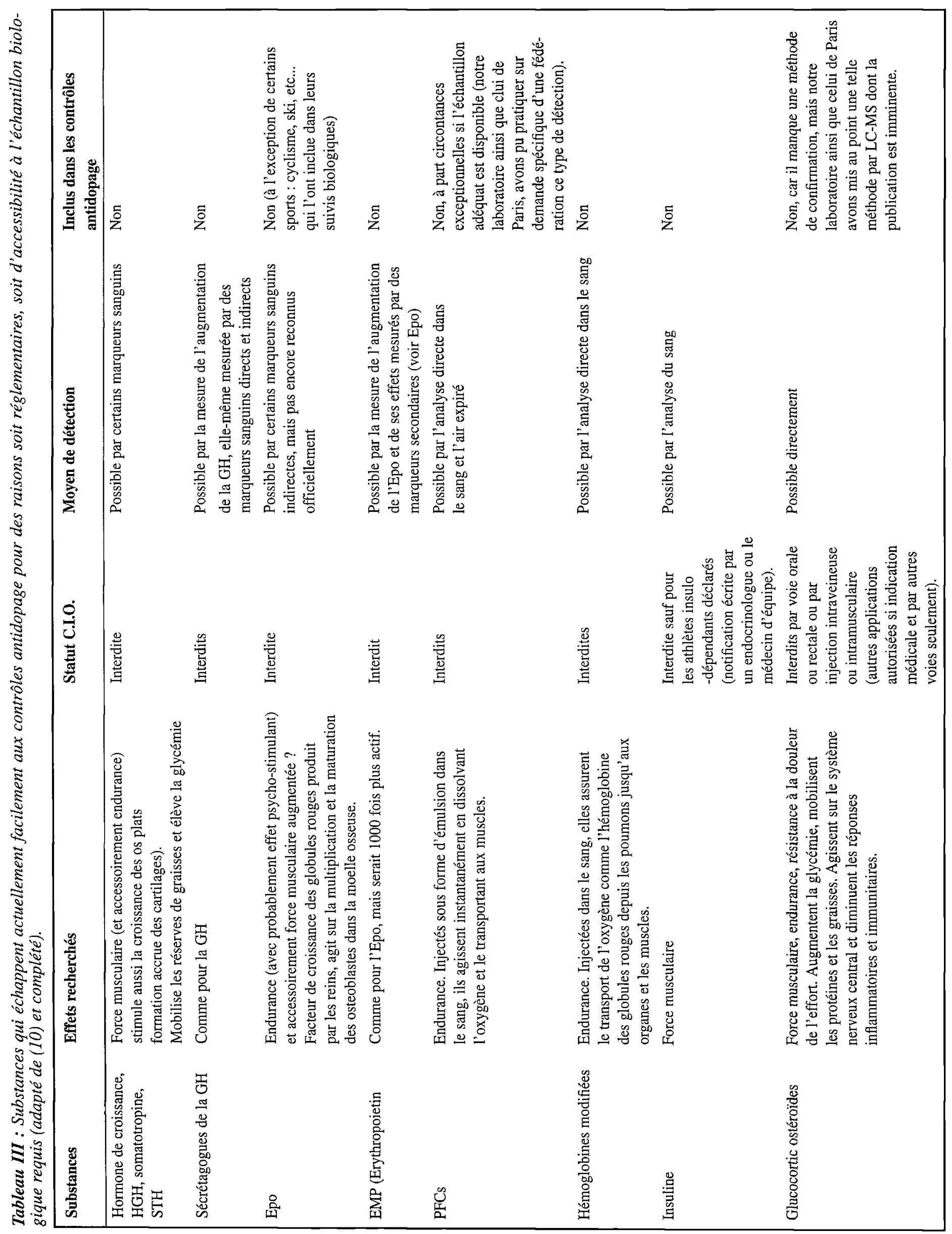




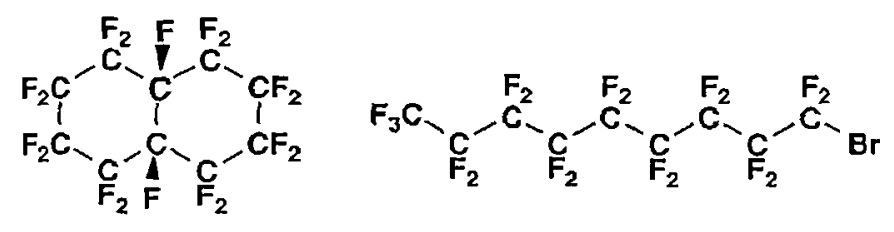

Figure 1 : Formule développée de deux PFCs, la Perfuorodecaline et le Perfuorooctylbromure, utilisés dans le cadre de la composition d'émulsions injectables destinées à l'usage clinique et en phase d'expérimentation.

quées par exemple par un exercice physique trop intensif et/ou trop prolongé. Les curarisants sont des molécules chimiques bien définies de type ammonium quaternaire qui exigent des techniques d'extraction (par formation de paires d'ions) et de détection particulières, comme, par exemple, la GC-MS, la LC-MS ou la LC-MS/MS (16). Ces méthodologies instrumentales ne sont pas encore fréquemment mises en cuvre dans les laboratoires de dépistage du dopage humain alors qu'elles sont beaucoup plus couramment employées dans les laboratoires de dépistage du dopage animal (en Europe pour les chevaux, en particulier) ou en toxicologie médico-légale (sur des matrices non conventionnelles, comme lors de l'analyse des cheveux, par exemple). Ces substances et/ou leurs métabolites ainsi que d'autres molécules polaires - sont dans ces conditions relativement facilement détectables dans l'urine.

3. Dans le même ordre d'idée, il existe une grande quantité de xénobiotiques qui n'ont jamais fait l'objet de tests extensifs et qui pourraient un jour être utilisées pour modifier les performances physiques. Il s'agit de l'immense corpus des molécules synthétisées par les industries pharmaceutiques pour la recherche de substances originales plus actives que les précédentes, mais qui, pour une raison ou une autre, n'ont jamais été mises sur le marché. Il existe certainement un stock important de chacune de ces substances, et à défaut d'y avoir accès, il ne doit pas être trop difficile de puiser dans les archives des brevets de nombreuses maisons pharmaceutiques pour y trouver des structures chimiques à partir desquelles un chimiste en mal d'imagination pourrait s'inspirer... De nouveau, puisqu'il s'agit dans ces conditions de substances étrangères, il ne devrait pas être trop compliqué de les détecter dans les urines.

4. Les laboratoires de dépistage antidopage adoptent des seuils de détection les plus bas possibles. On estime qu'en général, une dose de produit dopant de type stimulant peut être détectée plus de 60 heures après la prise pour le moins, alors que pour les anabolisants injectés par voie intramusculaires, les temps de détection peuvent dépasser 6 à 8 mois ! Or, il est imaginable d'absorber, non pas une large dose d'une seule substance active, mais des mini-doses de plusieurs composés ayant une activité pharmacologique globale similaire. L'effet résultant sera le même, mais l'analyse risquera bien de passer à côté de ce cocktail. Pour les substances exogènes, il faut donc être le plus sensible possible.

5. Enfin, au régime des substances exogènes, on peut également rattacher certains stéroïdes provenant du règne végétal ou du monde des insectes et des crustacés, par exemple. Les stérö̈des anabolisants ne semblent en particulier pas restreints au monde animal. Selon le Dr. Patrice Laure (17) des chercheurs russes auraient effectués des essais sur des sportifs avec des substances nommées "Ecdystéroïdes". Ces substances, au squelette chimique stéroïdien caractéristique de 27 à 29 atomes de carbone (Figure 2), contre 19 pour les androgènes, constituent jusqu'à $3 \%$ du poids sec de certaines plantes. Chez les insectes ou les crustacés, ces molécules auraient un rôle déterminant au moment de la mue, plus précisément en facilitant la synthèse de certaines protéines impliquées dans la formation de tissus. D'où l'idée de certains chercheurs que ces substances pourraient présenter des effets similaires chez les manmifères. Le monde des éleveurs de bétail s'est déjà intéressé à alimenter les animaux de fourrage enrichi de plantes à hautes teneurs en ecdystéroïdes. Toujours selon l'article du Dr. P. Laure, il existerait en Russie des concentrés d'ectystéroïdes tirés d'une fougère Leuzea carthomoides Iljin vendus sous le mon de Ecdisten qui provoqueraient des effets spectaculaires sur la performance physique. D'autre plantes sont également productrices de ces stéroïdes telles que le Suma (Pfaffia paniculata Martius) originaire d'Amazonie brésilienne ou du genre Rhaponticum de la famille des Composées. L'extrait du Suma est offert sous le nom de "Russian Secret"sur Internet, naturellement! Diverses préparations purifiées et concentrées seraient déjà disponibles dans plusieurs pays et contiendraient des taux variables d'écdystérone. La publicité qui leur est faite met surtout l'accent sur l'augmentation de muscles dans le cadre de la pratique des culturistes. Rien n'est connu à ce jour sur la innocuité de ces produits. Ces substances ne figurent pas expressément aujourd'hui sur la liste des produits prohibés, mais doivent être considérées comme dopantes, bien que la masse de publications scientifiques sur ce sujet spécifique démontrant leurs effets sur 1'homme soit particulièrement faible. En tout état de cause la détection de l'ecdystérone et de ses dérivés naturels ne pose pas de problème majeur pour le laboratoire, à part l'accessibilité aux substances de référence, les molécules mères et plus particulièrement les produits de leurs métabolites.

6. Profitant du libéralisme commercial de certains Etats, des laboratoires pharmaceutiques ont proposés à la vente toute une série de nouvelles molécules proches 


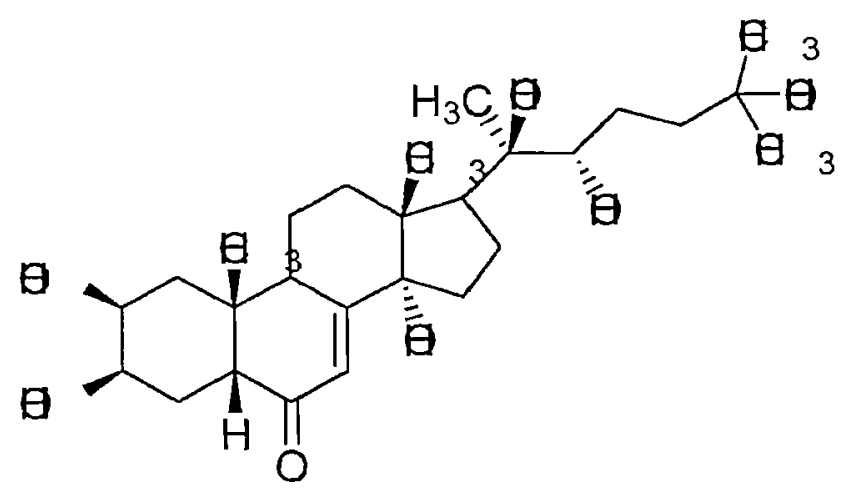

Figure 2 : Formule développée de la béta-ecdystérone (bEcdysone ou 20-Hydroxyecdysone), molécule de PM $=480$; C27 H44 O7 ; Merck Index $1 I^{\text {ème }}$ édition $N^{\circ} 3465$, considérée comme "hormone stimulant la croissance et la mue des insectes et présentant des effets anabolisants chez l'homme sans activité androgène".

de la testostérone. Les plus connues à ce jour sont la 5-alpha-Dihydrotestostérone (DHT) ou la Dehydroépiandrostérone (DHEA) et l'androstérone. D'autres molécules dérivées de la nor-testostérone (nandrolone) sont produites et vendues légalement aux U.S.A. telles la nor-androstènedione ou la nor-androstènediol. Ces pro-hormones (considérées donc aux Etats Unis comme des produits diététiques ou des compléments alimentaires) donnent l'illusion d'être des produits autorisés alors qu'ils sont totalement prohibés par le C.I.O. Leur détection ne pose pas de problème pour les laboratoires car lors du dépistage, les produits communs de leurs métabolismes respectifs seront recherchés. Il existe pourtant encore d'autres molécules le long de la chaîne de biosynthèse de la testostérone qui pourraient un jour devenir disponibles. Leur détection ne devrait alors pas poser de problèmes supplémentaires aux laboratoires sauf si on leur demande de spécifier la nature exacte du produit absorbé - indication qu'il n'est pas possible pour l'instant de donner.

7. Du côté des hormones peptidiques, on parle de la mise au point de petites molécules de synthèse capables, si elles atteignent le tissu cible, d'amener l'organisme à produire plus d'hormone naturelle. Plusieurs de ces petits peptides ont déjà été produits et testés avec des résultats impressionnants. Ils semblent dépourvus d'effets secondaires et peuvent être administrés par voie orale ou en spray nasal. Appelés "sécrétagogues ou peptides mimétiques" ou "Elicitators" en anglais, ils ne sont que faiblement absorbés par l'organisme sous leur forme galénique actuelle. A ce jour tant l'hGH (voir Tableau 3) que l'Epo (par le biais du "Erythropoietin Mimetic Peptide") semblent être les premières hormones cibles. Il semblerait que la détection de ces petits peptides ne poserait pas de difficultés majeures car, selon Romano Deghengi de Europeptide, lui-même à l'origine de la synthèse de l'hexaréline et cité par T. Souccar (10), "ces produits sont très facilement détectables dans le plasma et les urines, d'autant qu'il faut les prendre quotidiennement pour obtenir un effet durable sur la sécrétion de l'hormone". Encore faut-il que les laboratoires recherchent ces substances ou leurs métabolites de façon spécifique, ce qui n'est pas le cas aujourd'hui.

\section{Produits naturels}

La problématique de la détection des produits endogènes est plus complexe que celle des xénobiotiques. La difficulté principale de démontrer une absorption de substance prohibée réside dans la nécessité de distinguer la source naturelle de l'apport externe en substance identique, analyse non réalisable par les techniques conventionnelles de GC-MS ou LC-MS. A ce propos, l'introduction récente de la chromatographie gazeuse couplée à la mesure en continu du rapport isotopique du Carbone par spectrométrie de masse (GC-C-IRMS) a prouvé son efficacité dans la caractérisation du dopage à la testostérone et apparemment par quelques autres stéroïdes endogènes tels la DHT ou la DHEA (18). D'autres applications sont en cours de préparation. Mais il existe des composés plus complexes, fabriquées par les techniques recombinantes et qui sont les copies conformes des molécules fabriqués par le corps humain. Les techniques d'analyse ne sont pas capables de différencier pour le moment la substance synthétique de la naturelle. C'est le cas notamment pour l'hGH et l'Epo. A propos de la détection de l'hGH, nous avions proposé en 1997 déjà de pratiquer des contrôles urinaires hors compétition en fixant un seuil à 100 ng GH/L (11).. Cette proposition n'a malheureusement pas été mise en pratique, car le projet de recherche conjoint de l'Union européenne et du C.I.O. promettait des mesures plus efficaces basées sur l'analyse sanguine. Aux dernières nouvelles, il semble qu'une solution acceptable selon les normes du C.I.O. (efficacité proche du $100 \%$ ) ne soit pas encore trouvée. Mais, ces deux hormones peptidiques ne sont pas les seules en cause. D'autres molécules ont été synthétisées par technique recombinante, qui sont aujourd'hui disponibles en quantités suffisantes pour approfondir leurs mérites en thérapeutique. Ces préparations sont à peine étudiées sur le plan pharmacologique que déjà certains imaginent leur utilisation pour modifier les performances physiques, alors que rien n'est assuré quant à leur innocuité ou leur efficacité. En voici la description sommaire :

1. Dans le cadre du développement de produits de substitution du sang humain (cf. Tableau 4), un certain nombre d'hémoglobines modifiées ont été produites. Ces substituts des globules rouges doivent, tout comme les émulsions de PFCs, avoir la capacité de transporter suffisamment d'oxygène vers les tissus périphériques 
Tableau IV : État de la recherche appliquée pour certains produits potentiellement dopants du futur (adapté de 10, 12 et 20).

\begin{tabular}{|c|c|c|c|}
\hline Substance & Société & Nom du produit & Statut \\
\hline \multirow[t]{5}{*}{ Émulsions de PCF } & Green Cross, Japon & Fluosol(Perfluorodecaline) & Commercialisé mais abandonné dès 1990 \\
\hline & Green Cross, Japon & Oxypherol (Perfluorotributylamine) & Pas destiné à un usage clinique \\
\hline & Perftoran & Perftoran (Perfluorodecaline) & Commercialisé en Russie dès 1996 \\
\hline & Alliance & Oxygent (Perfluoro-octylbromure) & Essais cliniques de phase III \\
\hline & Hemagen/Baxter & Oxyfluor (Perfluorodichlorooctane) & Développement abandonné \\
\hline \multirow[t]{7}{*}{ Hémoglobine modifiée } & Baxter & $\begin{array}{l}\text { HemAssist. } \\
\text { Hémoglobine humaine réticulée }\end{array}$ & Développement arrêté en 1998 \\
\hline & Biopure & $\begin{array}{l}\text { Hemopure } \\
\text { Hémoglobine bovine réticulée } \\
\text { (polymérisée par la glutaraldéhyde) }\end{array}$ & Essais cliniques de phase II \\
\hline & Apex Bioscience & $\begin{array}{l}\text { PHP } \\
\text { (Hb humaine pyridoxylée et } \\
\text { conjuguée à du polyoxyethylène }\end{array}$ & Essais cliniques de phase II \\
\hline & Enzon & $\begin{array}{l}\text { PEG - Hemoglogin } \\
\text { (Hémoglobine bovine réticuléel } \\
\text { conjuguée à du polyethylène glycol) }\end{array}$ & Essais cliniques en phase III \\
\hline & Hémosol & $\begin{array}{l}\text { Hemolink } \\
\text { (Hémoglobine humaine polymérisée } \\
\text { à du o-raffinose) }\end{array}$ & Essais cliniques en phase III \\
\hline & Somatogen (Baxter) & $\begin{array}{l}\text { Optro } \\
\text { Hémoglobine humaine recombinante } \\
\text { di-alpha }\end{array}$ & Essais cliniques en phase II \\
\hline & Northfield & $\begin{array}{l}\text { Polyheme } \\
\text { Hémoglobine humaine réticulée }\end{array}$ & Essais cliniques en phase III \\
\hline \multirow[t]{5}{*}{$\begin{array}{l}\text { Sécrétagogues de } \\
\text { l'hormone de croissance }\end{array}$} & Merck & MK 677 & $\begin{array}{l}\text { Programme abandonné mais produit } \\
\text { disponible sur internet }\end{array}$ \\
\hline & Kaken & GHRP-2 & Essais cliniques en phase III \\
\hline & Novo Nordisk & NN 703 & Essais cliniques en phase II \\
\hline & Novo Nordisk & Ipamoréline & Programme abandonné \\
\hline & Mediolanum & Hexaréline & Essais cliniques en phase II \\
\hline
\end{tabular}

pendant un laps de temps relativement long. Cliniquement, on envisage l'emploi de tels produits lorsque l'organisme a perdu plus de la moitié de son volume normal de sang et que les tissus vitaux commencent à souffrir nettement du manque d'oxygénation. Ces transporteurs d'oxygène liés à l'hémoglobine sont de deux types : les premiers, non cellulaires, sont constitués d'hémoglobine reconstituée, croisée, recombinante ou polymérisée, alors que les seconds, cellulaires, sont dits "formé d'hémoglobine encapsulée", c'est-à-dire que les molécules d'hémoglobine purifiée sont contenues dans des vésicules de phospholipides (19). Aucun de ces produits n'est prêt pour une utilisation systématique en clinique car ils produisent apparemment encore trop d'effets secondaires incontrôlables. Vu les similitudes d'effets entre les PFCs et ces produits à base d'hémoglobine, certains ont vu des possibilités nouvelles de dopage.
Sur le plan de la détection de ces préparations non cellulaires, ce n'est pas sur la molécule d'hémoglobine elle-même qu'il conviendrait de baser les tests de dépistage puisqu'on se trouve en face de la même molécule. Cependant, il semble à première vue qu'il ne devrait pas être trop compliqué de caractériser dans l'urine certains produits de dégradation, mais c'est certainement l'examen direct du sang qui autoriserait une détection immédiate : la partie surnageant d'un sang centrifugé devrait présenter une coloration rouge accentuée et bien spécifique de l'hémoglobine si bien qu'à part une petite centrifugeuse - qui pourrait même fonctionner à main - il ne serait pas nécessaire en principe de faire appel à de l'instrumentation complexe. Quant aux préparations d'hémoglobine encapsulée, il est probable qu'un automate de comptage des cellules du sang de type Coulter, par exemple, devrait pouvoir être paramétré pour mettre le doigt sur l'administration 
d'une telle préparation pendant les quelques heures où elle est encore efficace. Mais il est certain qu'actuellement, les substances émises par de telles préparations ne sont pas recherchées par les laboratoires accrédités.

2. Parmi les produits obtenus par technique recombinante, il faut citer l'"Insuline Growth Factor One"(IGF-1) et l'Interleukine-3 (IL3). L'IGF-1 remplacerait l'hGH dont on parle de la caractérisation prochaine dans les tests de dépistage du dopage. De son côté l'IL3, facteur de croissance qui intervient sur les cellules souches de la moelle osseuse serait déjà détourné de la clinique par certains milieux sportifs pour une action bénéfique similaire à celle de l'Epo. Enfin le "Stem Cell Factor" (SCF), qui active aussi les cellules souches de la moelle, serait déjà utilisé en milieu sportif avant même que son autorisation de mise sur le marché n'ait été délivrée par les autorités sanitaires. Bien entendu, aucun laboratoire ne peut prétendre être en mesure de caractériser aujourd'hui de telles pratiques.

3. Subtilement, certains ont émis l'idée qu'on pouvait envisager l'utilisation d'autres facteurs de croissance liés à un organe ou un tissu particulier (vaisseaux, neurones, tissus osseux ou tendineux, etc.) ou d'orienter la biochimie tissulaire en fonction de l'effort prévu. Ces molécules de type protéique sont facilement synthétisées par génie génétique. La leptine est, par exemple, responsable de la régulation du stockage des acides gras. L'idée serait d'une part d'appliquer in vivo certains facteurs de croissance pour forcer des cellules à synthétiser d'autres facteurs de croissance plus complexes et qu'il est difficile de faire parvenir in situ. Tout l'art serait de faire parvenir les substances au bon endroit. Certains envisagent la voie d'implants souscutanés ou de véritables petites fabriques cellulaires encapsulées dans des membranes artificielles (20) qui empêchent leur rejet par l'organisme hôte.

D'autre part, on pourrait également imaginer la réparation de tissus abîmés par l'application in vitro de ces facteurs à des prélèvements pour permettre leur prolifération. Une fois que la quantité de tissus produits est suffisante, le manipulateur pourrait ré-introduire la masse de tissus dans la place qui souffrait d'un tel manque et restaurer la fonction complète (21). De là à imaginer que des techniciens modifient ainsi le fonctionnement d'un organe pour coller plus précisément aux exigences sportives de l'individu, il y a un pas à franchir dont on ne sait quand il pourrait intervenir.

4. Enfin, il ne faut négliger le potentiel qu'apporteraient la biologie génétique et la thérapie génique (22) : par l'acquis des connaissances fines de l'activation ou la suppression d'un gène, comme c'est le cas pour certaines maladies congénitales (par exemple : la maladie de Duschenne - correspondant à une myopathie héréditaire - par l'intermédiaire de laquelle on pourrait connaître les gènes et protéines impliqués dans cette maladie musculaire), on devrait déboucher sur une meilleure connaissance des mécanismes de régulation et les utiliser au profit non seulement des malades, mais les détourner de leur finalité première pour les appliquer dans le contexte de l'amélioration sportive. Poussant le raisonnement encore plus loin, on pourrait imaginer qu'en procédant à l'étude approfondie du gène du nanisme (maladie de Laron) - caractérisé par la mutation sur le gène codant de l'hGH, on arrive à en savoir suffisamment pour inverser son rôle et agir sur les gènes pour augmenter la production endogène de hGH. La question de l'expression ou la répression de ce gène devra bien sûr être résolue dans la foulée.....La tentation est donc grande d'imaginer de jouer avec l'ADN des sportifs. A partir de telles scénarios, on imagine mal le rôle du laboratoire de dépistage du dopage sous sa forme actuelle et il est probable qu'une profonde mutation sera nécessaire.

\section{Conclusion}

Tout un éventail de potentialité d'agents dopants a été présenté. Certains d'entre eux formeront un obstacle sérieux à la réussite des contrôles du dopage sportif du future. Il y en aura certainement d'autres encore. L'évolution des techniques de détection du dopage ces dernières années démontre heureusement une accélération sensible, l'éthique médicale ne doit plus être bafouée et l'imagination des tricheurs, si elle n'a plus de limites, doit absolument être freinée. Espérons aussi que certaines perspectives esquissées plus haut ne se réaliseront jamais. Nous devons donc conserver une vigilance constante pour nous adapter aux tendances qui se font jour. Les laboratoires accrédités devront compter sur l'aide de leurs collègues de la toxicologie pour faire face à la multitude des nouveaux problèmes qui immanquablement se poseront. Préparons-nous ensemble à relever ce défi si nous voulons que le sport reste une activité humaine équilibrée, noble et enrichissante. 


\section{Références}

1. Bourg J.-F, L'industrie du sport. Alternatives Économiques 1999 ; 172, 26-9.

2. Rivier L. Une zone grise dans le dopage des sportifs : les substances ergogènes. Médecine et Hygiène $1999 ; 57$ : 2034-41.

3. Bourg J.-F., Gouguet J.-J. Analyse Économique du sport. Paris : PUF. 1998.

4. Comité International Olympique Code Antidopage du Mouvement olympique. Lausanne : CIO. 1999.

5. Reynold J.E.F. (Edt.) Martindale, The extra Pharmacopoeia, 31st Edition, London : Royal Pharmaceutical Society 1996: 2739.

6. de Merode A. Lettre du 10 décembre envoyée aux Laboratoires accrédités par le CIO, Membres de la commission médicale du CIO, Membres du CIO, Membres honoraires, Comités Nationaux Olympiques, Fédérations internationales olympiques, Fédérations Internationales reconnues, Comités d'organisation des Jeux Olympiques et Organisations reconnues, 1999.

7. Mueller R.K. Communication personnelle, 1999.

8. Swiss Pharmaceutical Society Index Nominum, International Drug Directory, 17th Edition. Stuttgart : Medpharm 2000:1932.

9. Conconi F., Casoni I., Manfredini F., Mazzoni G., Grazzi G., Guglielmini C., Ballarin E., Borsetto C., Buzzoni D., Guerra G., Ricci G., Dapporto M., Rigolin F. Detection of erythropoietin administration in sports. In : P. Hemmersbach et K.I. Birkeland (Edts.) Blood samples in doping control. Oslo : On Demand Publishing 1994 : 133-140.

10. Souccar T. La course aux nouveaux dopants. Sciences et Avenir 1999 ; Août : 62-7.

11. Saugy M., Cardis C., Schweizer C., Veuthey J.-L., Rivier $\mathrm{L}$. The detection of human growth hormone doping in urine : Out of competition tests are necessary. J. Chromatogr. B 1996; 687 : 201-11.

12. Riess J.G., Keipert P.E. Update on Perfluorocarbonbased oxygen delivery systems. In "Blood substitutes - Present and future perspectives, E. Tsuchida (Edt.) Amsterdam : Elsevier 1998 ; 91-102.
13. Lowe K.C. Fluorocarbon emulsions as blood substitutes. In "Blood substitutes - Present and future perspectives, E. Tsuchida (Edt.) Amsterdam : Elsevier 1998 ; 327-38.

14. Audran M., Krafft M.-P., de Ceaurriz J., Mathurin J.-C., Sicart M.-T., Marion B., Fabre F., Bressolle F. Assay method for the perfluorooctyl bromide (peflubron) in rat blood by gas chromatographymass spectrometry. J. Chromatogr. B 1999 ; 734 : 267-76.

15. Rivier L., Mellingsaeter T., Jensen E. Detection of perfluorocarbons by head space gas chromatography - mass spectrometry in blood samples. J. Anal. Tox. 2000 ; (envoyé pour publication).

16. Giroud C., Augsburger M., Guignard N., Mahdad A., Procureur A., Steiger C., Rivier L. Les 48 victimes du drame de la secte de l'Ordre du Temple solaire : notre stratégie pour l'analyse toxicologique systématique. Actes du symposium STRATOX II, A. Traqui (Edt.) Strasbourg : IUML 1995 : 243-8.

17. Laure P. Les stéroïdes oubliés. Sport et Vie 1999 ; $55,66-7$.

18. Ueki M., Okano M. Doping with naturally occuring steroids. J. Toxicol - Toxin reviews $1999 ; 18$ : 177 195.

19. Tsuchida E. Perspective of Blood substitues. In "Blood substitutes - Present and future perspectives, E. Tsuchida (Edt.) Amsterdam : Elsevier $1998 ; 1-14$.

20. Winslow R.M. The role of blood substitutes in emerging healthcare systems. In "Blood substitutes - Present and future perspectives, E. Tsuchida (Edt.) Amsterdam : Elsevier 1998 ; 15-32.

21. Modex Therapeutics SA, Rue du Bugnon 27, 1005 Lausanne, Suisse.

22. Benoit-Browaeys D., Monnier E. La dope du futur. Science et Vie Hors Série $1999 ; 206$ : 48-52. 